\title{
A Frequency-Dependent LOD-FDTD Method and Its Application to the Analyses of Plasmonic Waveguide Devices
}

\author{
Jun Shibayama, Member, IEEE, Akifumi Nomura, Ryoji Ando, Junji Yamauchi, Senior Member, IEEE, and
} Hisamatsu Nakano, Fellow, IEEE

\begin{abstract}
Detailed frequency-dependent formulations are presented for several efficient locally one-dimensional finite-difference time-domain methods (LOD-FDTDs) based on the recursive convolution (RC), piecewise linear RC (PLRC), trapezoidal RC (TRC), auxiliary differential equation, and $Z$ transform techniques. The performance of each technique is investigated through the analyses of surface plasmon waveguides, the dispersions of which are expressed by the Drude and Drude-Lorentz models. The simple TRC technique requiring a single convolution integral is found to offer the comparable accuracy to the PLRC technique with two convolution integrals. As an application, a plasmonic grating filter is studied using the TRC-LOD-FDTD. The use of an apodized and a chirped grating is found quite effective in reducing sidelobes in the transmission spectrum, maintaining a large bandgap. Furthermore, a plasmonic microcavity is analyzed, in which a defect section is introduced into a grating filter. Varying the air core width is shown to exhibit tunable properties of the resonance wavelength.
\end{abstract}

Index Terms-Alternating-direction implicit finite-difference time-domain method (ADI-FDTD), auxiliary differential equation (ADE), dispersive media, surface plasmon polariton (SPP).

\section{INTRODUCTION}

$\mathbf{M}$ ETAL-insulator-metal-type optical waveguides can allow a surface plasmon polariton (SPP) to propagate through subwavelength structures [1]-[5]. This fact has received much attention, since compact optical circuits may be realized. For the finite-difference time-domain (FDTD) analysis of these structures where the SPP is highly localized along the metal/insulator interface, extremely small spatial sampling widths are required to attain sufficient accuracy. Unfortunately, this gives rise to a long computational time when the traditional explicit FDTD is used due to a small time step determined by the Courant-Friedrich-Levy (CFL) condition [6]. The use of the implicit FDTD can circumvent this problem, since it is free from the CFL condition.

Manuscript received March 03, 2009; revised May 16, 2009. Current version published December 04, 2009. This work was supported in part by MEXT, Grant-in-Aid for Scientific Research (c) (19560355).

J. Shibayama, R. Ando, J. Yamauchi, and H. Nakano are with the Faculty of Engineering, Hosei University, Koganei, Tokyo 184-8584, Japan (e-mail: shiba@hosei.ac.jp; ryoji.ando.fb@gs-eng.hosei.ac.jp; j.yma@k.hosei.ac.jp; hymat@hosei.ac.jp).

A. Nomura is with Japan Electron Optics Laboratory (JEOL) Co., Ltd., Haijima, Tokyo 196-8558, Japan (e-mail: anomura@ jeol.co.jp).

Digital Object Identifier 10.1109/JQE.2009.2024328
As an alternative to the alternating-direction implicit (ADI) FDTD [7], [8], we have proposed an implicit FDTD based on the locally one-dimensional (LOD) scheme [9]-[11]. The main advantage of the LOD-FDTD lies in a quite simple algorithm, maintaining the accuracy comparable to the ADI-FDTD. This fact has motivated researchers to improve and extend the LOD-FDTD, e.g., the development of low numerical dispersion schemes [12]-[14], the formulation of perfectly matched layers (PMLs) [15]-[17], and the application to three-dimensional (3-D) problems [18]-[21]. To analyze plasmonic devices in which the metal dispersion should be considered, we have developed several frequency-dependent LOD-FDTDs based on the recursive convolution (RC), piecewise linear RC (PLRC) [22], [23], trapezoidal RC (TRC) [24], auxiliary differential equation (ADE) [22], and $Z$ transform (ZT) [25] techniques. However, comparison among them has not yet been fully made, particularly for the analysis of plasmonic devices under the condition that the time step is beyond the CFL limit.

This paper describes a comparison of the above mentioned frequency-dependent LOD-FDTDs in a consistent manner, along with the traditional explicit FDTD. After presenting detailed formulations, not available in previous papers, we analyze the pulse propagation of the TM wave in the air core region sandwiched between the metal claddings. It is shown for both Drude and Drude-Lorentz models that the simple TRC technique with a single convolution integral provides the accuracy almost identical to the PLRC counterpart with two convolution integrals. For the Drude-Lorentz model, the ZT-LOD-FDTD allows to use a relatively large time step about ten times as large as that of the explicit FDTD. The CPU time of the LOD-FDTD is significantly reduced in comparison with the explicit FDTD, e.g., the time of the TRC-LOD-FDTD is reduced to $\simeq 30-50 \%$ of the explicit counterpart, maintaining almost the same accuracy.

As an application, a plasmonic grating filter is analyzed using the efficient TRC-LOD-FDTD. Note that in the conventional grating filter high sidelobes appear in the transmission spectrum. To suppress the sidelobes, the sine-shaped cell was introduced to the grating filter. Although the sidelobe level is reduced to some extent, the sidelobes still exist due to the sequence of the same cells. In addition, the sine-shaped cell reduces the contrast of the effective index in the grating section, giving rise to a narrow bandgap in the transmission spectrum. To address this issue, we introduce not only an apodized grating [26] but also a chirped grating into the filter. With each structure, the sidelobes 
are found to be successfully suppressed, while maintaining a large bandgap.

Final investigation is given to a plasmonic microcavity, in which a defect section is introduced into a grating filter. Taking advantage of the fact that the width of the air core region can be varied, we obtain tunable properties in the resonance wavelength. It is shown that the resonance wavelength remarkably shifts by more than $0.30 \mu \mathrm{m}$ with increasing the core width from $0.05 \mu \mathrm{m}$ to $0.2 \mu \mathrm{m}$.

\section{FREQUENCY-DEPENDENT LOD-FDTD FORMULATIONS}

\section{A. Dispersion Model}

The dispersion of a metal has frequently been taken into account using the Drude model. Note that the Drude model is generally applicable only to the near infrared region. In addition, the following Drude-Lorentz model [27]-[29] (with $P$ poles for the Lorentz function [30]) has also been used to account for the measured permittivities of $\mathrm{Au}$ and $\mathrm{Ag}$ over a wide spectrum from the visible to near infrared regions:

$$
\varepsilon_{r}(\omega)=\varepsilon_{\infty}+\frac{\omega_{\mathrm{D}}^{2}}{j \omega\left(\nu_{\mathrm{D}}+j \omega\right)}+\sum_{p=1}^{P} \frac{\Delta \varepsilon G_{p} \omega_{p}^{2}}{j \omega \nu_{p}+\omega_{p}^{2}-\omega^{2}}
$$

where $\varepsilon_{\infty}$ is the dielectric constant of the material at infinite frequency, $\omega$ is the angular frequency, $\omega_{\mathrm{D}}$ and $\omega_{p}$ are the electron plasma frequencies, $\nu_{\mathrm{D}}$ and $\nu_{p}$ are the effective electron collision frequencies, $G_{p}$ is the pole strength, and $\Delta \varepsilon$ is the weighting coefficient. Eliminating the last term in the right-hand side of (1) results in the Drude model.

In what follows, we present the LOD-FDTD formulations for the Drude-Lorentz model. Note that the equations for the Drude model can easily be obtained with the Lorentz terms being ignored in each formulation.

\section{B. Various RC Techniques}

The RC-based techniques are widely used to formulate the frequency-dependent FDTD, since the calculation of the convolution integral can be efficiently performed. Note that the techniques are applicable only to linear dispersive materials.

The $D-E$ relation in time domain is expressed as

$$
\begin{aligned}
\boldsymbol{D}(t) & =\varepsilon_{0} \varepsilon_{\infty} \boldsymbol{E}(t)+\varepsilon_{0} \int_{0}^{n \Delta t} \boldsymbol{E}(n \Delta t-\tau) \chi(\tau) d \tau \\
& \equiv \varepsilon_{0} \varepsilon_{\infty} \boldsymbol{E}(t)+\varepsilon_{0} \stackrel{\boldsymbol{P}}{\boldsymbol{P}(n \Delta t)}
\end{aligned}
$$

where $\varepsilon_{0}$ is the permittivity of free space, $\boldsymbol{P}$ is the linear polarization and $\chi(\omega)$ is susceptibility.

For the RC technique [30], $\boldsymbol{P}$ is approximated as

$$
\boldsymbol{P}(n \Delta t)=\sum_{m=0}^{n-1} \boldsymbol{E}^{n-m} \chi^{m}
$$

where

$$
\chi^{m}=\int_{m \Delta t}^{(m+1) \Delta t} \chi(\tau) d \tau
$$

in which the electric field is assumed constant over $\Delta t$.

For the PLRC technique [31], $\boldsymbol{P}$ is approximated as

$$
\boldsymbol{P}(n \Delta t)=\sum_{m=0}^{n-1}\left\{\boldsymbol{E}^{n-m} \chi^{m}+\left(\boldsymbol{E}^{n-m-1}-\boldsymbol{E}^{n-m}\right) \xi^{m}\right\}
$$

where

$$
\xi^{m}=\frac{1}{\Delta t} \int_{m \Delta t}^{(m+1) \Delta t}(\tau-m \Delta \tau) \chi(\tau) d \tau
$$

in which the electric field has piecewise linear functional dependence over $\Delta t$. Note that the term regarding $\xi^{m}$ appears compared with (3), requiring an additional calculation of the convolution integral. This formulation results in storage of electric field values from two time steps [31]-[33].

With the PLRC technique, we obtain the following equation of the LOD-FDTD for the TM wave (the normalized expression of field components is used throughout the formulations):

$$
\begin{aligned}
E_{x}^{\prime}= & E_{x}^{n} \\
E_{z}^{\prime}= & \frac{\varepsilon_{\infty}-\xi^{0}}{\varepsilon_{\infty}+\chi^{0}-\xi^{0}} E_{z}^{n}+\frac{1}{\varepsilon_{\infty}+\chi^{0}-\xi^{0}} \phi_{z}^{n} \\
& +\frac{c \Delta t}{2\left(\varepsilon_{\infty}+\chi^{0}-\xi^{0}\right)}\left(\frac{\partial H_{y}^{\prime}}{\partial x}+\frac{\partial H_{y}^{n-1 / 2}}{\partial x}\right) \\
\frac{H_{y}^{\prime}-H_{y}^{n-1 / 2}}{\Delta t / 2}= & c\left(\frac{\partial E_{z}^{\prime}}{\partial x}+\frac{\partial E_{z}^{n}}{\partial x}\right)
\end{aligned}
$$

for the first step and

$$
\begin{aligned}
E_{z}^{n+1}= & E_{z}^{\prime} \\
E_{x}^{n+1}= & \frac{\varepsilon_{\infty}-\xi^{0}}{\varepsilon_{\infty}+\chi^{0}-\xi^{0}} E_{x}^{\prime}+\frac{1}{\varepsilon_{\infty}+\chi^{0}-\xi^{0}} \phi_{x}^{n} \\
& -\frac{c \Delta t}{2\left(\varepsilon_{\infty}+\chi^{0}-\xi^{0}\right)} \\
& \times\left(\frac{\partial H_{y}^{n+1 / 2}}{\partial z}+\frac{\partial H_{y}^{\prime}}{\partial z}\right) \\
\frac{H_{y}^{n+1 / 2}-H_{y}^{\prime}}{\Delta t / 2}= & -c\left(\frac{\partial E_{x}^{n+1}}{\partial z}+\frac{\partial E_{x}^{\prime}}{\partial z}\right)
\end{aligned}
$$

for the second step, where $E^{\prime}$ and $H^{\prime}$ represent the intermediate fields, $c$ is the speed of light in a vacuum, $\chi^{0}=\chi_{\mathrm{D}}^{0}+$ $\sum_{p=1}^{P} \operatorname{Re}\left[\chi_{p}^{0}\right]$, and $\phi_{\delta}^{n}=\phi_{\mathrm{D}}^{n}+\sum_{p=1}^{P} \operatorname{Re}\left[\phi_{p}^{n}\right]$, in which $\delta=x$ or $z \cdot \phi_{\mathrm{D}}^{n}$ and $\phi_{p}^{n}$ are expressed as follows:

$$
\begin{aligned}
\phi_{\mathrm{D}}^{n} & =\left(\Delta \chi_{\mathrm{D}}^{0}-\Delta \xi_{\mathrm{D}}^{0}\right) E_{\delta}^{n}+\Delta \xi_{\mathrm{D}}^{0} E_{\delta}^{n-1}+e^{-\nu_{\mathrm{D}} \Delta t} \phi_{\mathrm{D}}^{n-1} \\
\phi_{p}^{n} & =\left(\Delta \chi_{p}^{0}-\Delta \xi_{p}^{0}\right) E_{\delta}^{n}+\Delta \xi_{p}^{0} E_{\delta}^{n-1}+e^{\gamma \Delta t} \phi_{p}^{n-1}
\end{aligned}
$$


where $\gamma=-\alpha+j \beta, \alpha=\nu_{p} / 2$, and $\beta=\sqrt{\omega_{p}^{2}-\alpha^{2}}$. The parameters used in the above formulation are given as

$$
\begin{aligned}
\chi_{\mathrm{D}}^{0} & =\frac{\omega_{\mathrm{D}}^{2}}{\nu_{\mathrm{D}}}\left\{\Delta t-\frac{1}{\nu_{\mathrm{D}}}\left(1-e^{-\nu_{\mathrm{D}} \Delta t}\right)\right\} \\
\chi_{p}^{0} & =-\frac{2 \Delta \varepsilon G_{p} \omega_{p}^{2}}{\gamma\left(\gamma-\gamma^{*}\right)}\left(1-e^{\gamma \Delta t}\right) \\
\Delta \chi_{\mathrm{D}}^{0} & =-\frac{\omega_{\mathrm{D}}^{2}}{\nu_{\mathrm{D}}^{2}}\left(1-e^{-\nu_{\mathrm{D}} \Delta t}\right)^{2} \\
\Delta \chi_{p}^{0} & =\chi_{p}^{0}\left(1-e^{\gamma \Delta t}\right) \\
\xi_{\mathrm{D}}^{0} & =\frac{\omega_{\mathrm{D}}^{2}}{\nu_{\mathrm{D}}}\left\{\frac{\Delta t}{2}-\frac{1}{\nu_{\mathrm{D}}^{2} \Delta t}\left(1-e^{-\nu_{\mathrm{D}} \Delta t}\right)+\frac{1}{\nu_{\mathrm{D}}} e^{-\nu_{\mathrm{D}} \Delta t}\right\} \\
\xi_{p}^{0} & =-j \frac{\Delta \varepsilon G_{p} \omega_{p}^{2}}{\gamma^{2} \beta \Delta t}\left\{(\gamma \Delta t-1) e^{\gamma \Delta t}+1\right\} \\
\Delta \xi_{\mathrm{D}}^{0} & =-\frac{\omega_{\mathrm{D}}^{2}}{\nu_{\mathrm{D}}^{2}}\left\{\frac{1}{\nu_{\mathrm{D}} \Delta t}\left(1-e^{-\nu_{\mathrm{D}} \Delta t}\right)^{2}\right. \\
\Delta \xi_{p}^{0} & =\xi_{p}^{0}\left(1-e^{\gamma \Delta t}\right) .
\end{aligned}
$$

In the first step, we substitute (5c) into (5b) and implicitly solve the resultant equation. Then, $(5 c)$ is explicitly solved. In the second step, the equations are calculated in the same way as in the first step. It should be noted that for the LOD-FDTD two implicit and two explicit equations are solved. As a result, the number of explicit equations to be solved is reduced, when compared with the ADI counterpart in which two implicit and four explicit equations should be solved. Comparison between (3) and (4) shows that neglecting the terms regarding $\xi$ in (5) and (6) results in the equations for the $\mathrm{RC}$ technique.

As an alternative, the TRC technique was developed for the frequency-dependent formulation [32], [33]. The advantage of the TRC technique lies in the fact that it requires only a single convolution integral maintaining the accuracy comparable to the PLRC counterpart with two convolution integrals. Although the TRC technique was applied to the Debye and Lorentz models in [32], [33], no attempt was made to apply it to the Drude model frequently used for the analysis of plasmonic devices. Recently, we have applied the TRC technique to the Drude-Lorentz model for a concise frequency-dependent formulation [24]. Since only the brief formulation has been presented in [24], we here derive detailed finite-difference equations of the TRC-based LODFDTD.

For the TRC technique, $\boldsymbol{P}$ is approximated using an average of the electric fields over two consecutive time steps in the following form:

$$
\boldsymbol{P}(n \Delta t)=\sum_{m=0}^{n-1} \frac{\boldsymbol{E}^{n-m}+\boldsymbol{E}^{n-m-1}}{2} \chi^{m} .
$$

Obviously, the TRC technique requires only a single convolution integral regarding $\chi^{m}$, as in the $\mathrm{RC}$ technique. As a result of the formulation similar to the RC procedure, we obtain the TRC-LOD-FDTD equations, in which (5b) and (6b) obtained from the PLRC technique are replaced, respectively, with the following (8) and (9):

$$
\begin{aligned}
E_{z}^{\prime}= & \frac{\varepsilon_{\infty}-\chi^{0} / 2}{\varepsilon_{\infty}+\chi^{0} / 2} E_{z}^{n}+\frac{1}{\varepsilon_{\infty}+\chi^{0} / 2} \phi_{z}^{n} \\
& +\frac{c \Delta t}{2\left(\varepsilon_{\infty}+\chi^{0} / 2\right)}\left(\frac{\partial H_{y}^{\prime}}{\partial x}+\frac{\partial H_{y}^{n-1 / 2}}{\partial x}\right) \\
E_{x}^{n+1}= & \frac{\varepsilon_{\infty}-\chi^{0} / 2}{\varepsilon_{\infty}+\chi^{0} / 2} E_{x}^{\prime}+\frac{1}{\varepsilon_{\infty}+\chi^{0} / 2} \phi_{x}^{n} \\
& -\frac{c \Delta t}{2\left(\varepsilon_{\infty}+\chi^{0} / 2\right)}\left(\frac{\partial H_{y}^{n+1 / 2}}{\partial z}+\frac{\partial H_{y}^{\prime}}{\partial z}\right)
\end{aligned}
$$

where $\phi_{\delta}^{n}=\phi_{\mathrm{D}}^{n}+\sum_{p=1}^{P} \operatorname{Re}\left[\phi_{p}^{n}\right]$, in which $\delta=x$ or $z . \phi_{\mathrm{D}}^{n}$ and $\phi_{p}^{n}$ are expressed as follows:

$$
\begin{aligned}
\phi_{\mathrm{D}}^{n} & =\frac{E_{\delta}^{n}+E_{\delta}^{n-1}}{2} \Delta \chi_{\mathrm{D}}^{0}+e^{-\nu_{\mathrm{D}} \Delta t} \phi_{\mathrm{D}}^{n-1} \\
\phi_{p}^{n} & =\frac{E_{\delta}^{n}+E_{\delta}^{n-1}}{2} \Delta \chi_{p}^{0}+e^{\gamma \Delta t} \phi_{p}^{n-1} .
\end{aligned}
$$

Clearly, the equations for the TRC-LOD-FDTD are simpler than those for the PLRC-LOD-FDTD. We can solve the TRC-LODFDTD equations as done in the PLRC-LOD-FDTD.

\section{ADE Technique}

If we want to avoid the calculations of the convolution integrals, we can resort to the ADE technique [34], [35], in which the $D-E$ relation in frequency domain is translated into that in time domain using the inverse Fourier transform.

The $E-H$ relation in frequency domain is expressed as

$$
\frac{j \omega \varepsilon_{r}(\omega)}{c} \boldsymbol{E}=\nabla \times \boldsymbol{H}
$$

After substituting (1) into (10) and applying the inverse Fourier transform to the resultant equation $(j \omega \rightarrow \partial / \partial t)$, we obtain

$$
\begin{aligned}
\varepsilon_{\infty} \frac{\partial \boldsymbol{E}}{\partial t}+\boldsymbol{J}+\sum_{p=1}^{P} \frac{\partial \boldsymbol{Q}_{p}}{\partial t} & =c \nabla \times \boldsymbol{H} \\
\frac{\partial \boldsymbol{J}}{\partial t}+\nu_{\mathrm{D}} \boldsymbol{J} & =\omega_{\mathrm{D}}^{2} \boldsymbol{E} \\
\frac{\partial^{2} \boldsymbol{Q}_{p}}{\partial t^{2}}+\nu_{p} \frac{\partial \boldsymbol{Q}_{p}}{\partial t}+\omega_{p}^{2} \boldsymbol{Q}_{p} & =\Delta \varepsilon G_{p} \omega_{p}^{2} \boldsymbol{E} .
\end{aligned}
$$

Discretizing the above equations with the LOD scheme leads to the equations for the first step as

$$
\begin{gathered}
\varepsilon_{\infty} \frac{E_{z}^{n+1}-E_{z}^{n}}{\Delta t / 2}+J_{z}^{n+1}+J_{z}^{n}+\sum_{p=1}^{P} \frac{Q_{p, z}^{n+1}-Q_{p, z}^{n}}{\Delta t / 2} \\
=c\left(\frac{\partial H_{y}^{\prime}}{\partial x}+\frac{\partial H_{y}^{n-1 / 2}}{\partial x}\right) \\
\frac{H_{y}^{\prime}-H_{y}^{n-1 / 2}}{\Delta t / 2}=c\left(\frac{\partial E_{z}^{n+1}}{\partial x}+\frac{\partial E_{z}^{n}}{\partial x}\right) \\
\frac{J_{z}^{n+1}-J_{z}^{n}}{\Delta t}+\nu_{\mathrm{D}} \frac{J_{z}^{n+1}+J_{z}^{n}}{2}=\omega_{\mathrm{D}}^{2} \frac{E_{z}^{n+1}+E_{z}^{n}}{2}
\end{gathered}
$$




$$
\begin{aligned}
& \frac{Q_{p, z}^{n+1}-2 Q_{p, z}^{n}-Q_{p, z}^{n-1}}{\Delta t^{2}}+\nu_{p} \frac{Q_{p, z}^{n+1}-Q_{p, z}^{n-1}}{2 \Delta t} \\
& \quad+\omega_{p}^{2} \frac{Q_{p, z}^{n+1}+Q_{p, z}^{n-1}}{2} \\
& =\Delta \varepsilon G_{p} \omega_{p}^{2} \frac{E_{z}^{n+1}+E_{z}^{n}}{2} .
\end{aligned}
$$

Here, we carefully choose the time step to be $\Delta t$ in (16) and (17), instead of $\Delta t / 2$ used in (14) and (15) [22]. This is due to the fact that splitting (16) and (17) is not required, since the intermediate fields are regarded as the fields at $n+1$ in the LOD procedure, as shown in (6a). Consequently, we can apply the standard central difference with $\Delta t$ to the discretization of (16) and (17). This also implies simpler implementation of the LOD algorithm, compared with the ADE-based ADI-FDTD [29] in which the intermediate fields should be calculated with (16) and (17) being split.

For the second step, we have

$$
\begin{aligned}
& \varepsilon_{\infty} \frac{E_{x}^{n+1}-E_{x}^{n}}{\Delta t / 2}+J_{x}^{n+1}+J_{x}^{n}+\sum_{p=1}^{P} \frac{Q_{p, x}^{n+1}-Q_{p, x}^{n}}{\Delta t / 2} \\
& =-c\left(\frac{\partial H_{y}^{n+1 / 2}}{\partial z}+\frac{\partial H_{y}^{\prime}}{\partial z}\right) \\
& \frac{H_{y}^{n+1 / 2}-H_{y}^{\prime}}{\Delta t / 2}=-c\left(\frac{\partial E_{x}^{n+1}}{\partial z}+\frac{\partial E_{x}^{n}}{\partial z}\right) \\
& \frac{J_{x}^{n+1}-J_{x}^{n}}{\Delta t}+\nu_{\mathrm{D}} \frac{J_{x}^{n+1}+J_{x}^{n}}{2}=\omega_{\mathrm{D}}^{2} \frac{E_{x}^{n+1}+E_{x}^{n}}{2} \\
& \frac{Q_{p, x}^{n+1}-2 Q_{p, x}^{n}-Q_{p, x}^{n-1}}{\Delta t^{2}}+\nu_{p} \frac{Q_{p, x}^{n+1}-Q_{p, x}^{n-1}}{2 \Delta t} \\
& \quad+\omega_{p}^{2} \frac{Q_{p, x}^{n+1}+Q_{p, x}^{n-1}}{2} \\
& =\Delta \varepsilon G_{p} \omega_{p}^{2} \frac{E_{x}^{n+1}+E_{x}^{n}}{2} .
\end{aligned}
$$

To solve the above equations, we first substitute (15)-(17) into (14) and implicitly solve $E_{z}^{n+1}$. Once $E_{z}^{n+1}$ is obtained, the other components are solved explicitly. The solutions for the second step are calculated similarly.

\section{Z Transforms (ZTs)}

Another way of avoiding the calculations of the convolution integrals is to use the ZTs [36]. In this technique, the complicated convolution integrals, treated in the RC-based techniques, can simply be reduced to algebraic equations, and the $D-E$ relation can be translated into finite-difference equations in a straightforward manner, compared with the ADE technique.

To apply the ZT theory, we rewrite (1) as

$$
\begin{aligned}
\varepsilon_{r}(\omega)=\varepsilon_{\infty}+\frac{\omega_{\mathrm{D}}^{2} / \nu_{\mathrm{D}}}{j \omega} & -\frac{\omega_{\mathrm{D}}^{2} / \nu_{\mathrm{D}}}{\nu_{\mathrm{D}}+j \omega} \\
& +\sum_{p=1}^{P} \frac{\Delta \varepsilon G_{p} \omega_{p}^{2} \beta / \beta}{\alpha^{2}+\beta^{2}+j 2 \alpha \omega+(j \omega)^{2}} .
\end{aligned}
$$

Taking the $Z$ transform of (22) and $\boldsymbol{D}(\omega)=\varepsilon_{r}(\omega) \boldsymbol{E}(\omega)$ (with the field normalization) yields the following equations in the $Z$ domain:

$$
\begin{aligned}
\boldsymbol{D}(z) & =\varepsilon_{\infty} \boldsymbol{E}(z)+z^{-1} \boldsymbol{M}(z)+z^{-1} \sum_{p=1}^{P} \boldsymbol{Q}_{p}(z) \\
\boldsymbol{M}(z) & =\frac{\frac{\omega_{\mathrm{D}}^{2} \Delta t}{\nu_{\mathrm{D}}}\left(1-e^{-\nu_{\mathrm{D}} \Delta t}\right)}{1-\left(1+e^{-\nu_{\mathrm{D}} \Delta t}\right) z^{-1}+e^{-\nu_{\mathrm{D}} \Delta t} z^{-2}} \boldsymbol{E}(z) \\
\boldsymbol{Q}_{p}(z) & =\frac{\frac{\Delta \varepsilon G_{p} \omega_{p}^{2} \Delta t}{\beta} e^{-\alpha \Delta t} \sin (\beta \Delta t)}{1-2 e^{-\alpha \Delta t} \cos (\beta \Delta t) z^{-1}+e^{-2 \alpha \Delta t} z^{-2}} \boldsymbol{E}(z)
\end{aligned}
$$

in which $\Delta t$ and $z^{-l}$ represent the time period and the delay of $l$ time periods, respectively. We readily translate the above into the finite-difference equations in the $Z$ domain as

$$
\begin{aligned}
\boldsymbol{E}^{n+1}= & \frac{1}{\varepsilon_{\infty}} \boldsymbol{D}^{n+1}-\frac{1}{\varepsilon_{\infty}} \boldsymbol{M}^{n}-\frac{1}{\varepsilon_{\infty}} \sum_{p=1}^{P} \boldsymbol{Q}_{p}^{n} \\
\boldsymbol{M}^{n+1}= & \left(1+e^{-\nu_{\mathrm{D}} \Delta t}\right) \boldsymbol{M}^{n}-e^{-\nu_{\mathrm{D}} \Delta t} \boldsymbol{M}^{n-1} \\
& +\frac{\omega_{\mathrm{D}}^{2} \Delta t}{\nu_{\mathrm{D}}}\left(1-e^{-\nu_{\mathrm{D}} \Delta t}\right) \boldsymbol{E}^{n+1} \\
\boldsymbol{Q}_{p}^{n+1}= & 2 e^{-\alpha \Delta t} \cos (\beta \Delta t) \boldsymbol{Q}_{p}^{n}-e^{-2 \alpha \Delta t} \boldsymbol{Q}_{p}^{n-1} \\
& +\frac{\Delta \varepsilon G_{p} \omega_{p}^{2} \Delta t}{\beta} e^{-\alpha \Delta t} \sin (\beta \Delta t) \boldsymbol{E}^{n+1} .
\end{aligned}
$$

Note that the above equations regarding the $D-E$ relation are analytically derived without adopting the finite-difference approximation used in the ADE technique.

We now solve (23)-(25), combined with the following standard LOD-FDTD equations:

$$
\begin{aligned}
\frac{D_{z}^{n+1}-D_{z}^{n}}{\Delta t / 2} & =c\left(\frac{\partial H_{y}^{\prime}}{\partial x}+\frac{\partial H_{y}^{n-1 / 2}}{\partial x}\right) \\
\frac{H_{y}^{\prime}-H_{y}^{n-1 / 2}}{\Delta t / 2} & =c\left(\frac{\partial E_{z}^{n+1}}{\partial x}+\frac{\partial E_{z}^{n}}{\partial x}\right)
\end{aligned}
$$

for the first step. Using only the $z$ components in (23)-(25), we substitute (23) and (27) into (26) and implicitly solve the resultant tridiagonal equations, and then explicitly solve (23)-(25) and (27). Note that the relation $E_{z}^{n+1}=E_{z}^{\prime}$ or $D_{z}^{n+1}=D_{z}^{\prime}$, as shown in (6a), is used in the above implementation. The equations for the second step are

$$
\begin{aligned}
\frac{D_{x}^{n+1}-D_{x}^{n}}{\Delta t / 2} & =-c\left(\frac{\partial H_{y}^{n+1 / 2}}{\partial z}+\frac{\partial H_{y}^{\prime}}{\partial z}\right) \\
\frac{H_{y}^{n+1 / 2}-H_{y}^{\prime}}{\Delta t / 2} & =-c\left(\frac{\partial E_{x}^{n+1}}{\partial z}+\frac{\partial E_{x}^{n}}{\partial z}\right)
\end{aligned}
$$

where the relation $E_{x}^{\prime}=E_{z}^{n}$ or $D_{x}^{\prime}=D_{x}^{n}$, as shown in (5a), is used, which are similarly solved using only the $x$ components in (23)-(25). 


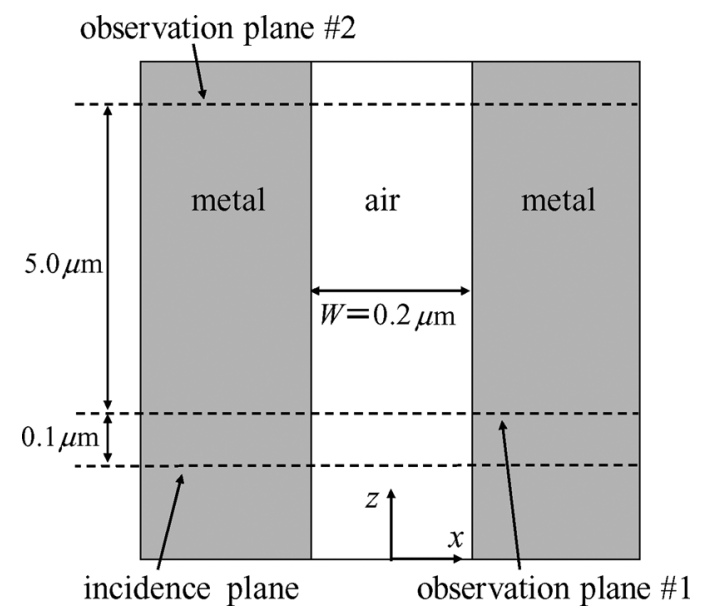

Fig. 1. Configuration of a metal cladding optical waveguide.

\section{COMPARATIVE STUDY}

We investigate the numerical accuracy and computational efficiency of each frequency-dependent LOD-FDTD through analyzing the optical waveguide shown in Fig. 1. Due to the symmetry of the waveguide, only half the section $(x>0)$ is analyzed, in which the computational window size is $0.3 \mu \mathrm{m} \times 10 \mu \mathrm{m}$ in the $x$ and $z$ directions, respectively. The sampling widths are $\Delta x=0.005 \mu \mathrm{m}$ and $\Delta z=0.004 \mu \mathrm{m}$. The upper limit of the CFL condition is defined as $\Delta t_{\mathrm{CFL}}(=0.0102 \mathrm{fs})$, and the ratio of $\Delta t / \Delta t_{\mathrm{CFL}}$ as CFL number (CFLN). The TM pulse wave is launched at the incidence plane (see Fig. 1), which consists of the eigenmode field in the $x$ direction and the Gaussian profile with a $1 / e$ full-width of $1 \mu \mathrm{m}$ in the $z$ direction. The pulse is illuminated using the one-way excitation scheme, so that no field propagates toward the $-z$ direction. Since the computational window is chosen large enough in the $z$ direction, the calculation finishes before the pulse propagating in the $+z$ direction reaches the computational window edge. Therefore, no specific absorbing boundary condition is employed. The wavelength characteristic of the transmission coefficient is calculated from the ratio between the discrete Fourier transforms of the incident pulse observed at \#1 and the transmitted pulse at \#2 after a propagation length of $z=5 \mu \mathrm{m}$.

Note that the treatment of the interface between a metal and a dielectric material is quite important in obtaining an accurate numerical result [37], [38]. Throughout this paper, we adopt the averaged permittivity between a metal and air along the interface on which the electric field component is placed [24], [37].

\section{A. Drude Model}

In the first example, we chose the metal to be Ag, the dispersion of which is determined by the Drude model in the near infrared region [4]: $\varepsilon_{\infty}=3.7, \omega_{\mathrm{D}}=9.1 \mathrm{eV}$, and $\nu_{\mathrm{D}}=0.018 \mathrm{eV}$.

Fig. 2 shows the transmission coefficient versus CFLN at the center wavelength of the incident pulse $(1.55 \mu \mathrm{m})$. Taking advantage of the unconditional stability, we use the LOD-FDTD for CFLN $\geq 1$. For CFLN $\leq 1$, the traditional explicit FDTD is used. For CFLN $=1$ the identical results are obtained from both LOD-FDTD and explicit FDTD, validating

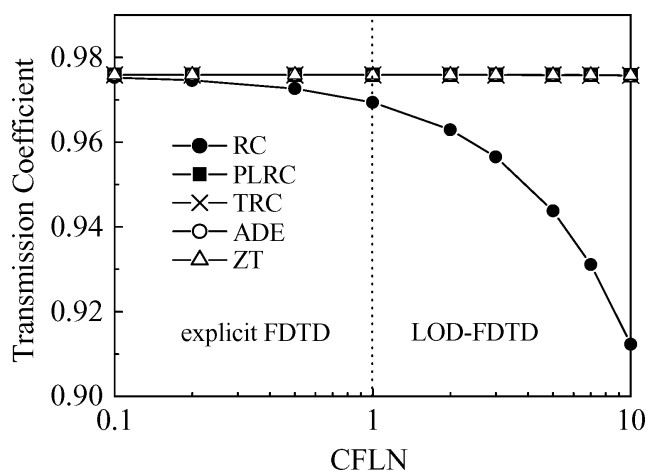

Fig. 2. Transmission coefficient versus CFLN for the Ag cladding expressed with the Drude model.

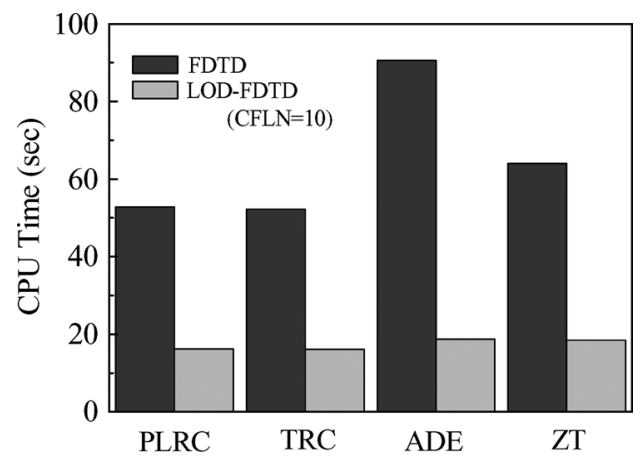

Fig. 3. CPU time for the Drude model.

the LOD-FDTD. It is noteworthy that the TRC technique offers almost the same results obtained from the PLRC, ADE and ZT techniques, regardless of the solution with a single convolution integral. The RC technique works only when CFLN is chosen quite small. For reference, we analyze the same waveguide using the frequency-domain eigenmode solver based on the Yee-mesh-based beam-propagation method [39], providing a transmission coefficient of 0.97585 . For CFLN $=1$, the errors of the LOD-FDTD results are calculated to be of the order of $10^{-3}$, while even for CFLN $=10$, they are of $10^{-2}$. As a result, we can employ a relatively large CFLN up to $\simeq 10$ for the LOD-FDTD.

The CPU time is presented in Fig. 3, where a PC with Core2 Quad processor $(2.66 \mathrm{GHz})$ is used. CFLN $=10$ is adopted for the LOD-FDTD calculations, in which the time of the RC technique is not shown due to the unacceptable results found in Fig. 2. It is seen that the CPU time of the ADE-based explicit FDTD is longer than the times of the other explicit methods. This is due to a number of arithmetic operations when the electric fields are calculated with ADEs (the CPU time may be reduced with an elaborate algorithm [40]). Fortunately, the time of the ADE-LOD-FDTD is the same level as the times of the other LOD-FDTDs. The relative CPU time of each LOD-FDTD to that of each explicit FDTD is as follows: $30 \%, 30 \%, 20 \%$, and $28 \%$ for the PLRC, TRC, ADE, and ZT techniques, respectively, indicating the high efficiency of the LOD-FDTD.

The memory requirement for each FDTD is illustrated in Fig. 4. In general, the implicit LOD scheme requires larger memory than the explicit scheme owing to the solution of 


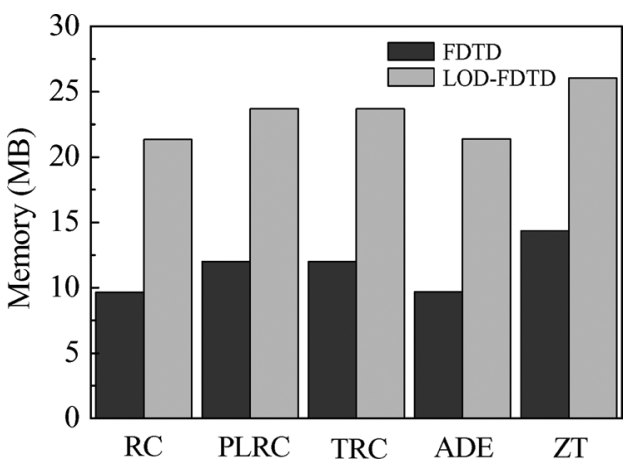

Fig. 4. Memory requirement for the Drude model.

a tridiagonal system of linear equations. Since the RC and ADE techniques need only the field values from a single time step, their memory requirements are smaller than the PLRC, TRC and ZT counterparts. For the ZT technique, the memory requirement is slightly large compared with the others, due to some auxiliary variables.

In the application in Section IV, we will use the simple TRCLOD-FDTD, due to its acceptable accuracy, less CPU time, and moderate memory requirement.

\section{B. Drude-Lorentz Model}

In the second example, we investigate Au in the visible region, the dispersion of which is accurately described with the Drude-Lorentz model. This is motivated by the fact that $\mathrm{Au}$ is frequently applied to the thin metal layer of the surface plasmon resonance sensor operated in the visible region. The parameters are $\varepsilon_{\infty}=5.9673, \omega_{\mathrm{D}} / 2 \pi=2113.6 \mathrm{THz}, \omega_{1} / 2 \pi=$ $650.07 \mathrm{THz}, \nu_{\mathrm{D}} / 2 \pi=15.92 \mathrm{THz}, \nu_{1} / 2 \pi=104.86 \mathrm{THz}$, and $\Delta \varepsilon=1.09$ [27], [29], which corresponds to $P=1$, so that $G_{1}=1$ in (1) [30].

Fig. 5 depicts the transmission coefficient versus CFLN at a center wavelength of $0.7 \mu \mathrm{m}$. For the explicit FDTDs (CFLN $\leq$ $1)$, there exists no difference in accuracy among the PLRC, TRC, ADE, and ZT techniques. It is interesting to note, however, that the difference can be seen with the LOD-FDTD being used for CFLN $\geq 1$. To investigate the accuracy in more detail, we display the expanded figure for the LOD-FDTD in Fig. 5(b). For reference, also included are the eigenmode solution (0.76451) and the value with $0.5 \%$ error from the solution, both of which are indicated by the dashed lines. It is seen that the ZT technique attains higher accuracy, resulting from the analytic derivation of the equation solved, to which no finite-difference approximation is applied. Although the PLRC and TRC techniques provide almost the same accuracy, careful investigation reveals that the former is slightly better than the latter. This is probably because for the former even the electric fields over $\Delta t$ are evaluated using the integration in addition to the electric fields on the two consecutive sampling times, while for the latter only the electric fields on the two consecutive sampling times are evaluated. Slightly less accurate results of the ADE technique for a large CFLN may be caused by the accuracy degradation in the finite-difference approximation of the temporal second derivative in (17) and (21).

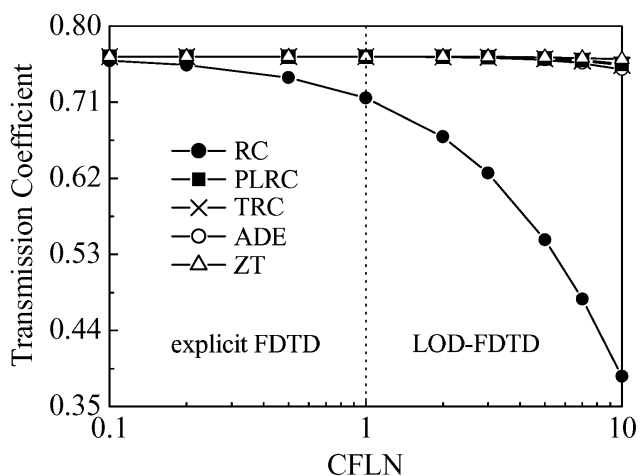

(a)

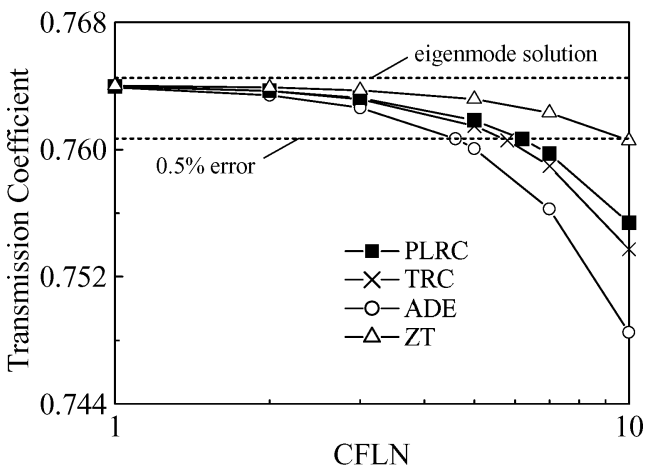

(b)

Fig. 5. Transmission coefficient versus CFLN for the Au cladding expressed with the Drude-Lorentz model.



Fig. 6. CPU time for the Drude-Lorentz model.

Here, we mention the computational efficiency of the LODFDTD, when the $0.5 \%$ error is accepted in the transmission coefficient. In this case, CFLNs can be extended to 6.2, 5.8, 4.6, and 10 for the PLRC, TRC, ADE, and ZT techniques, respectively, as shown in Fig. 5(b). Fig. 6 shows the CPU time for each method. It can be seen that the ZT-LOD-FDTD is slightly faster than the other techniques because of a large CFLN. When compared with the explicit FDTD, the CPU times of the LODFDTD are reduced to $48 \%, 52 \%, 40 \%$, and $25 \%$, respectively, for the PLRC, TRC, ADE, and ZT techniques. Fig. 7 shows the memory requirement. Since the field values from two time steps are required, the memory is almost the same among the PLRC, TRC, and ADE techniques. As in the case of Fig. 4, the ZT requires marginally larger memory than the others.

As seen in [27], for Au in the visible region there exists the strong dispersion of the imaginary part of the permittivity, which 


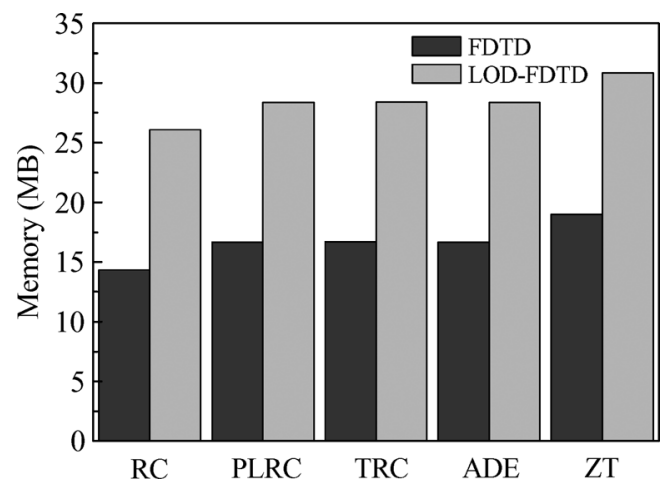

Fig. 7. Memory requirement for the Drude-Lorentz model.

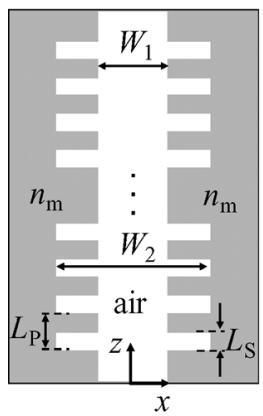

(a)

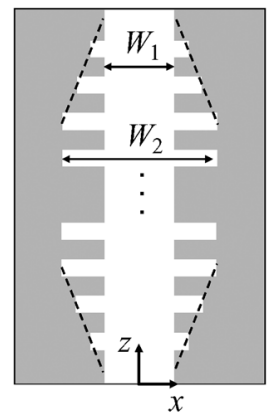

(b)

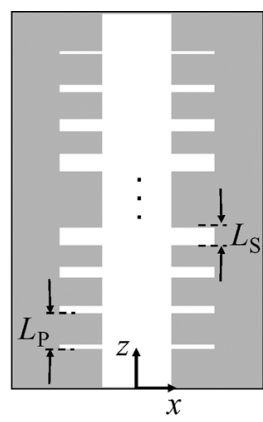

(c)
Fig. 8. Configurations of several plasmonic waveguide gratings. (a) Conventional; (b) apodized; (c) chirped.

is taken into account with the Lorentz term. It follows that a large CFLN cannot be used, unless the effect of the strong dispersion is accurately incorporated into the resultant equation to be solved. From this aspect, the ZT technique is expected to offer the use of a relatively large CFLN, since the equations considering the dispersion is accurately derived in an analytic manner without a finite-difference approximation.

\section{APPLICATION}

\section{A. Analysis of Plasmonic Waveguide Grating Filter}

The plasmonic grating filter [4], [5], formed by a periodic variation of the insulator width of the plasmonic waveguide, is one of the basic building blocks for small size plasmonic circuits. Note that in the conventional filter high sidelobes appear in the transmission spectrum. Here, we introduce an apodized and a chirped grating into the filter. As mentioned in Section III, we use the TRC-LOD-FDTD to characterize the modified grating filters.

Fig. 8(a) illustrates the configurations of the conventional filter, in which the configuration parameters are as follows: $W_{1}=0.1 \mu \mathrm{m}, W_{2}=0.2 \mu \mathrm{m}, L_{\mathrm{P}}=0.66 \mu \mathrm{m}$, $L_{\mathrm{S}}=0.292 \mu \mathrm{m}$, and the number of periods is 19 . The metal is $\mathrm{Ag}$, the material parameters of which are the same as those used in Section III-A. To absorb the outgoing waves, we impose the PML at the edges of the computational window. We adopt CFLN $=10$ throughout the application, where there is no stability problem of the PML.

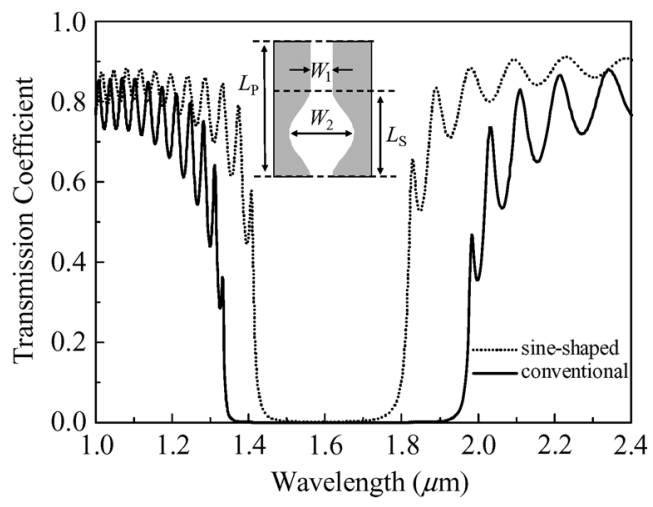

Fig. 9. Transmission coefficient for the conventional filter.

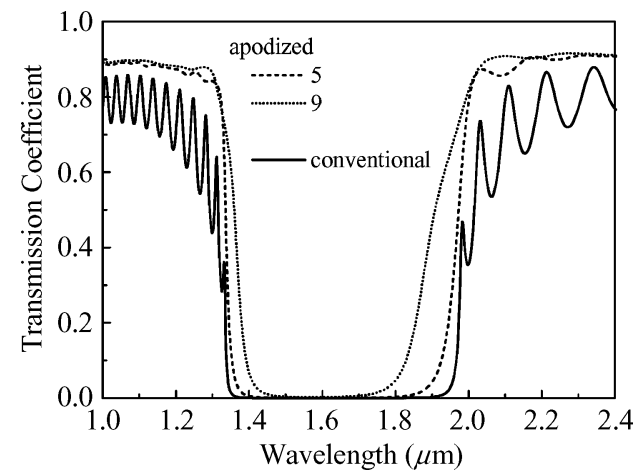

Fig. 10. Transmission coefficient for the apodized filter.

The transmission coefficient of the conventional filter is shown in Fig. 9 (solid line), where the high sidelobes can be seen. The sine-shaped cell, shown in the inset of Fig. 9, is used to suppress the sidelobes [4], and the coefficient is shown in Fig. 9 (dotted line). Although the sidelobes are suppressed with the sine-shaped cell in some degree, they still remain due to the sequence of the same cells. In addition, the reduction of the index contrast in the grating section gives rise to a narrow bandgap, compared with the conventional filter.

To address this issue, we introduce the apodization into the filter, as depicted in Fig. 8(b). Fig. 10 shows the transmission coefficient of the apodized filter, where we apply the linear apodization to the 5 or 9 periods at both input and output ports. For the 5 periods, the sidelobes are successfully suppressed, maintaining a large bandgap. In addition, the sidelobes are further suppressed as the number of apodized periods is increased, while the bandgap becomes slightly narrow. Hence, there should be an adequate number of apodized periods.

The first sidelobe level and the band width versus the number of apodized periods are quantitatively presented in Fig. 11, in which the band width is defined as the band where the transmission coefficient becomes under $-30 \mathrm{~dB}$. In Fig. 11, as the number of periods is increased, the sidelobe is suppressed and the band width is reduced, e.g., for the filter with 5 periods, the first sidelobe level is successfully suppressed to about $-1.5 \mathrm{~dB}$, while maintaining a relatively wide bandgap almost identical to the conventional filter. On the other hand, the effect of the sidelobe suppression is also expected with the use of the chirped grating. The results using the linear chirped grating shown in 


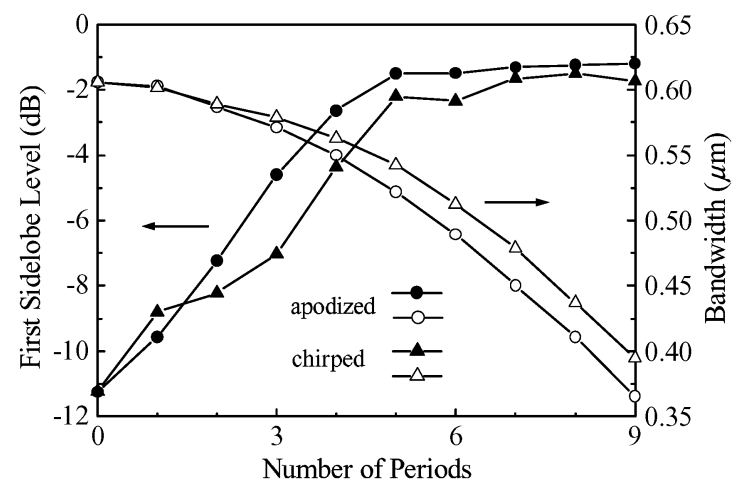

Fig. 11. First sidelobe level and band width.

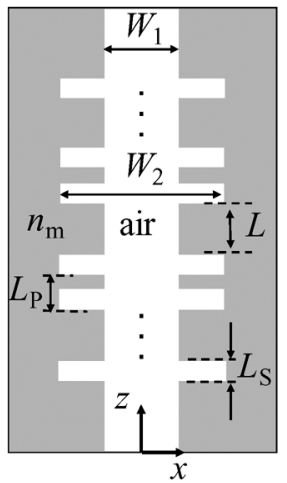

(a)

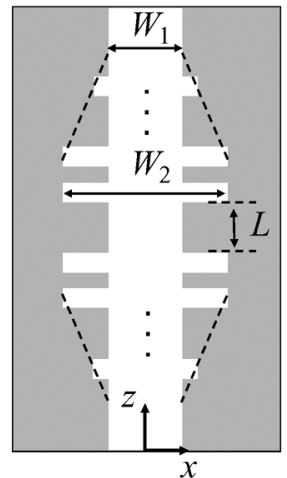

(b)
Fig. 12. Configurations of plasmonic microcavities. (a) Conventional; (b) apodized.

Fig. 8(c) are also included in Fig. 11. As in the case of the apodized grating, the sidelobe suppression is observed whereas the band width is reduced with the number of chirped periods. Consequently, the use of an apodized and a chirped grating is effective in reducing the sidelobe, with a large bandgap being retained.

\section{B. Analysis of Plasmonic Microcavities}

Finally, we investigate a plasmonic microcavity in which a defect section is introduced into the grating discussed above. Figs. 12(a) and (b) show, respectively, the conventional microcavity and the apodized microcavity for reduced sidelobes. The defect is $L=0.688 \mu \mathrm{m}$ in length and the number of gratings is 18. The other structural and material parameters are the same as those used in Fig. 8.

The transmission spectra are shown in Fig. 13, in which the linear apodization is applied to the 5 or 8 periods at both input and output ports of the microcavity. It can be seen that the transmission peak appears at $\lambda=1.6 \mu \mathrm{m}$ in the bandgap ranging from $1.3 \mu \mathrm{m}$ to $2.0 \mu \mathrm{m}$. As in the case of the grating, the apodization leads to a reduction of the sidelobes, when compared with the conventional microcavity.

Notice that the core width of the cavity can be varied, since the core is a hollow structure. The variation of the core width results in the effective index change of the waveguide region. This is expected to exhibit tunable properties of the resonance wavelength, which is closely related to a tunable hollow waveguide

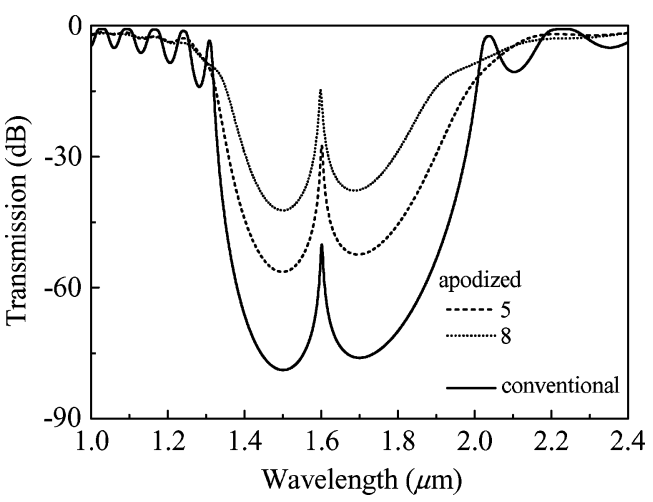

Fig. 13. Transmission.

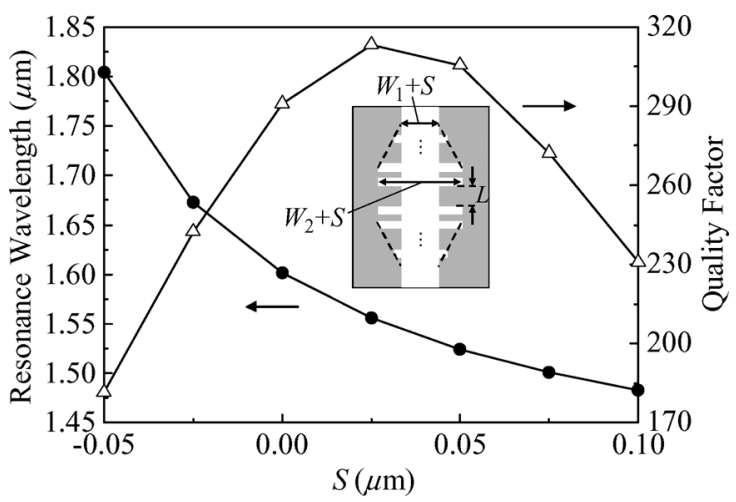

Fig. 14. Resonance frequency and Q factor versus $S$.

Bragg grating [41]. We are therefore motivated to examine the variation of the resonance wavelength for a variable core width, with the grating depth being fixed.

Fig. 14 depicts the resonance wavelength and quality factor versus $S$ defined in the inset, in which the 5 periods at both input and output ports are apodized. The quality factor is calculated from $\lambda_{0} / \Delta \lambda$ in which $\lambda_{0}$ and $\Delta \lambda$ are the central resonance wavelength and the full width at half maximum of the defect mode, respectively [3]. In the quality factor, a local maximum can be seen at $S=0.025 \mu \mathrm{m}$. This is where the resonance wavelength approximately coincides with the local minimum of the transmission spectrum of the grating. More importantly, the resonance wavelength is found to monotonically shift toward shorter wavelengths as $S$ is increased. A remarkable wavelength shift of more than $0.30 \mu \mathrm{m}$ is obtained for $S$ ranging from $-0.05 \mu \mathrm{m}$ to $0.1 \mu \mathrm{m}$.

\section{CONCLUSION}

We have assessed the performance of several frequency-dependent LOD-FDTDs based on the RC, PLRC, TRC, ADE, and ZT techniques. It is found that the simple TRC technique requiring only a single convolution integral offers the accuracy comparable to the PLRC counterpart with two convolution integrals for both Drude and Drude-Lorentz models. When the dispersion is strong, the ZT-LOD-FDTD allows us to use a large time step, since the equations considering the dispersion are analytically derived without a finite-difference approximation. The CPU times of the LOD-FDTDs are significantly reduced to 
$20 \%-50 \%$ of those of the traditional explicit FDTDs. The comparative study provides guidelines on the choice of an appropriate method for a practical application. We have also studied a plasmonic waveguide grating filter and a plasmonic microcavity. For the reduction of the sidelobes of the transmission coefficient, apodized and chirped gratings are found to be effective for both filter and cavity. Taking advantage of the hollow structure of the cavity, we show that tunable properties of the resonance wavelength can be obtained with varying the air core width. Left for future studies are the analyses of a coupling problem between a plasmonic and a dielectric waveguide, and of three-dimensional structures.

\section{REFERENCES}

[1] K. Tanaka and M. Tanaka, "Simulations of nanometric optical circuits based on surface plasmon polariton gap waveguide," Appl. Phys. Lett., vol. 82, no. 8, pp. 1158-1160, Feb. 2003.

[2] J. Takahara and F. Kusunoki, "Guiding and nanofocusing of two-dimensional optical beam for nanooptical integrated circuits," IEICE Trans. Electron., vol. E90-C, no. 1, pp. 87-94, Jan. 2007.

[3] A. Hossieni and Y. Massoud, "A low-loss metal-insulator-metal plasmonic bragg reflector," Opt. Express, vol. 14, no. 23, pp. 11318-11323, Nov. 2006.

[4] Z. Han, E. Forsberg, and S. He, "Surface plasmon Bragg gratings formed in metal-insulator-metal waveguides," IEEE Photon. Technol. Lett., vol. 19, no. 2, pp. 91-93, Jan. 2007.

[5] L. Yuan and Y. Y. Lu, "A recursive-doubling Dirichlet-to-Neumann-map method for periodic waveguides," J. Lightw. Technol., vol. 25, no. 11, pp. 3649-3656, Nov. 2007.

[6] A. Taflove and S. C. Hagness, Computational Electrodynamics: The Finite-Difference Time-Domain Method, 3rd ed. Norwood, MA: Artech House, 2005.

[7] T. Namiki, "A new FDTD algorithm based on alternating-direction implicit method," IEEE Trans. Microw. Theory Tech., vol. 47, no. 10, pp. 2003-2007, Oct. 1999.

[8] F. H. Zheng, Z. Z. Chen, and J. Z. Zhang, "A finite-difference time-domain method without the Courant stability conditions," IEEE Microw. Guided Wave Lett., vol. 9, no. 11, pp. 441-443, Nov. 1999.

[9] J. Shibayama, M. Muraki, J. Yamauchi, and H. Nakano, "Efficient implicit FDTD algorithm based on locally one-dimensional scheme," Electron. Lett., vol. 41, no. 19, pp. 1046-1047, Sep. 2005.

[10] J. Shibayama, M. Muraki, R. Takahashi, J. Yamauchi, and H. Nakano, "Performance evaluation of several implicit FDTD methods for optical waveguide analyses," J. Lightw. Technol., vol. 24, no. 6, pp. 2465-2472, Jun. 2006.

[11] J. Shibayama, B. Murakami, J. Yamauchi, and H. Nakano, "LOD-BOR-FDTD algorithm for efficient analysis of circularly symmetric structures," IEEE Microw. Wireless Compon. Lett., vol. 19, no. 2, pp. 56-58, Feb. 2009.

[12] E. Li, I. Ahmed, and R. Vahldieck, "Numerical dispersion analysis with an improved LOD-FDTD method," IEEE Microw. Wireless Compon. Lett., vol. 17, no. 5, pp. 319-321, May 2007.

[13] K.-Y. Jung and F. L. Teixeira, "An iterative unconditionally stable LOD-FDTD method," IEEE Microw. Wireless Compon. Lett., vol. 18, no. 2, pp. 76-78, Feb. 2008.

[14] F. Liang, G. Wang, and W. Ding, "Low numerical dispersion locally one-dimensional FDTD method based on compact higher-order scheme," Microw. Opt. Technol. Lett., vol. 50, no. 11, pp. 2783-2787, Nov. 2008.

[15] V. E. do Nascimento, B.-H. V. Borges, and F. L. Teixeira, "Split-field PML implementations for the unconditionally stable LOD-FDTD method," IEEE Microw. Wireless Compon. Lett., vol. 16, no. 7, pp. 398-400, Jul. 2006.

[16] O. Ramadan, "Unsplit field implicit PML algorithm for complex envelope dispersive LOD-FDTD simulations," Electron. Lett., vol. 43, no. 5, pp. 267-268, Mar. 2007.

[17] I. Ahmed, E. Li, and K. Krohne, "Convolutional perfectly matched layer for an unconditionally stable LOD-FDTD method," IEEE Microw. Wireless Compon. Lett., vol. 17, no. 12, pp. 816-818, Dec. 2007.

[18] E. L. Tan, "Unconditionally stable LOD-FDTD method for 3-D Maxwell's equations," IEEE Microw. Wireless Compon. Lett., vol. 17, no. 2, pp. 85-87, Feb. 2007.
[19] E. L. Tan, "Fundamental schemes for efficient unconditionally stable implicit finite-difference time-domain methods," IEEE Trans. Antennas Propagat., vol. 56, no. 1, pp. 170-177, Jan. 2008.

[20] I. Ahmed, E. K. Chua, E. P. Li, and Z. Chen, "Development of the three-dimensional unconditionally stable LOD-FDTD method," IEEE Trans. Antennas Propagat., vol. 56, no. 11, pp. 3596-3600, Nov. 2008.

[21] Q. F. Liu, Z. Z. Chen, and W. Y. Yin, "An arbitrary-order LOD-FDTD method and its stability and numerical dispersion," IEEE Trans. Antennas Propag., vol. 57, no. 8, pp. 2409-2417, Aug. 2009.

[22] J. Shibayama, R. Takahashi, J. Yamauchi, and H. Nakano, "Frequencydependent LOD-FDTD implementations for dispersive media," Electron. Lett., vol. 42, no. 19, pp. 1084-1086, Sep. 2006.

[23] J. Shibayama, R. Takahashi, J. Yamauchi, and H. Nakano, "Frequencydependent locally one-dimensional FDTD implementation with a combined dispersion model for the analysis of surface plasmon waveguides," IEEE Photon. Technol. Lett., vol. 20, no. 10, pp. 824-826, May 2008.

[24] J. Shibayama, R. Ando, A. Nomura, J. Yamauchi, and H. Nakano, "Simple trapezoidal recursive convolution technique for the frequencydependent FDTD analysis of a Drude-Lorentz model," IEEE Photon. Technol. Lett., vol. 21, no. 2, pp. 100-102, Jan. 15, 2009.

[25] J. Shibayama, R. Takahashi, A. Nomura, J. Yamauchi, and H. Nakano, "Concise frequency-dependent formulation for LOD-FDTD method using $Z$ transforms," Electron. Lett., vol. 44, no. 16, pp. 949-950, Jul. 2008.

[26] J. Shibayama, A. Nomura, R. Takahashi, J. Yamauchi, and H. Nakano, "Study of the sidelobe suppression in a plasmonic waveguide grating filter using the LOD-FDTD method," presented at the Integr. Photon. Nanophoton. Res. Appl., Boston, MA, Jul. 2008, IMB6, unpublished.

[27] A. Vial, A.-S. Grimault, D. Macías, D. Barchiesi, and M. L. de la Chapelle, "Improved analytical fit of gold dispersion: Application to the modeling of extinction spectra with a finite-difference time-domain method," Phys. Rev. B, vol. 71, pp. 085416-1-085416-7, Feb. 2005.

[28] T. Yamaguchi and T. Hinata, "Optical near-field analysis of spherical metals: Application of the FDTD method combined with the ADE method," Opt. Express, vol. 15, no. 18, pp. 11481-11491, Sep. 2007.

[29] K.-Y. Jung and F. L. Teixeira, "Multispecies ADI-FDTD algorithm for nanoscale three-dimensional photonic metallic structures," IEEE Photon. Technol. Lett., vol. 19, no. 8, pp. 586-588, Apr. 2007.

[30] R. J. Luebbers and F. Hunsberger, "FDTD for $N$ th-order dispersive media," IEEE Trans. Antennas Propag., vol. 40, no. 11, pp. 1297-1301, Nov. 1992.

[31] D. F. Kelley and R. J. Luebbers, "Piecewise linear recursive convolution for dispersive media using FDTD," IEEE Trans. Antennas Propag., vol. 44, no. 6, pp. 792-797, Jun. 1996.

[32] R. Siushansian and J. LoVetri, "A comparison of numerical techniques for modeling electromagnetic dispersive media," IEEE Microw. Guided Wave Lett., vol. 5, no. 12, pp. 426-428, Dec. 1995.

[33] R. Siushansian and J. LoVetri, "Efficient evaluation of convolution integrals arising in FDTD formulations of electromagnetic dispersive media," J. Electromag. Waves Appli., vol. 11, no. 1, pp. 101-117, 1997.

[34] T. Kashiwa and I. Fukai, "A treatment by FD-TD method of dispersive characteristics associated with electronic polarization," Microw. Opt. Technol. Lett., vol. 3, no. 6, pp. 203-205, Jun. 1990.

[35] R. M. Joseph, S. C. Hagness, and A. Taflove, "Direct time integration of Maxwell equations in linear dispersive media with absorption for scattering and propagation of femtosecond electromagnetic pulses," Opt. Lett., vol. 16, no. 18, pp. 1412-1414, May 1991.

[36] D. M. Sullivan, "Frequency-dependent FDTD methods using $Z$ Transforms," IEEE Trans. Antennas Propag., vol. 40, no. 10, pp. 1223-1230, Oct. 1992.

[37] Y. Zhao, P. Belov, and Y. Hao, "Accurate modeling of the optical properties of left-handed media using a finite-difference time-domain method," Phys. Rev. E, vol. 75, no. 3, pp. 037602-1-037602-4, Mar. 2007.

[38] T. Arima and T. Uno, "Boundary treatment method of dispersive materials analysis by FDTD method," (in Japanese) Trans. IEICE, vol. J91-B, no. 9, pp. 1066-1068, Sep. 2008.

[39] J. Yamauchi, T. Mugita, and H. Nakano, "Implicit Yee-mesh-based finite-difference full-vectorial beam-propagation method," J. Lightw. Technol., vol. 23, no. 5, pp. 1947-1955, May 2005.

[40] Y. Takayama and W. Klaus, "Reinterpretation of the auxiliary differential equation method for FDTD," IEEE Microw. Wireless Compon. Lett., vol. 12, no. 3, pp. 102-104, Mar. 2002.

[41] Y. Sakurai and F. Koyama, "Tunable hollow waveguide distributed Bragg reflectors with variable air core," Opt. Express, vol. 12, no. 13, pp. 2851-2856, Jun. 2004. 


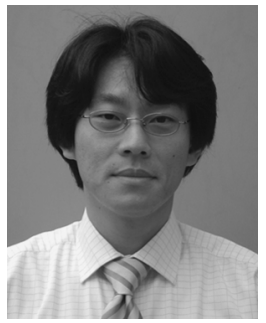

Jun Shibayama (M'03) was born in Kashiwa, Japan, on July 1, 1969. He received the B.E., M.E., and Dr.E. degrees from Hosei University, Tokyo, Japan, in 1993, 1995, and 2001, respectively.

From 1995 to 1999 , he worked at Opto-Technology Laboratory (currently FITEL Photonics Laboratory), Furukawa Electric Co., Ltd., Ichihara, Chiba, Japan. From 1999 to 2009, he served as an Assistant at Hosei University. Now, he is a Lecturer at Hosei. His research interests include the numerical analysis of electromagnetic problems.

Dr. Shibayama is a member of the Optical Society of America and the Institute of Electronics, Information and Communication Engineers (IEICE) of Japan.

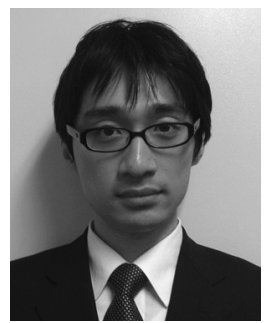

Akifumi Nomura was born in Shimane, Japan, on October 9, 1984. He received the B.E. and M.E degrees from Hosei University, Tokyo, Japan, in 2007 and 2009, respectively. In 2009, he joined Japan Electron Optics Laboratory (JEOL) Co., Ltd.

Mr. Nomura is a member of the Institute of Electronics, Information and Communication Engineers (IEICE) of Japan.

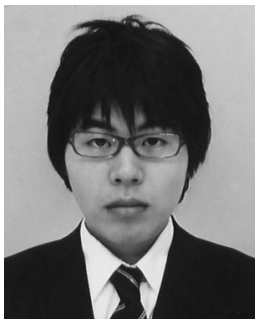

Ryoji Ando was born in Aichi, Japan, on September 25, 1985. He received the B.E. degree from Hosei University, Tokyo, Japan, in 2009 and is currently pursuing the M.E. degree.

Mr. Ando is a member of the Institute of Electronics, Information and Communication Engineers (IEICE) of Japan.

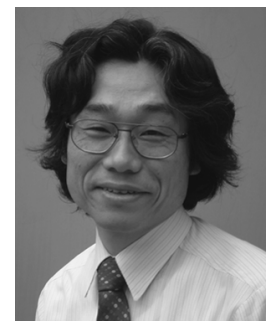

Junji Yamauchi (M'85-SM'08) was born in Nagoya, Japan, on August 23, 1953. He received the B.E., M.E., and Dr.E. degrees from Hosei University, Tokyo, Japan, in 1976, 1978, and 1982, respectively.

From 1984 to 1988 , he served as a Lecturer in the Electrical Engineering Department of Tokyo Metropolitan Technical College. Since 1988, he has been a member of the faculty of Hosei University, where he is now a Professor. His research interests include optical waveguides and circularly polarized antennas. $\mathrm{He}$ is the author of Propagating Beam Analysis of Optical Waveguides (Baldock, Hertfordshire, U.K.: Research Studies Press, 2003).

Dr. Yamauchi is a member of the Optical Society of America and the Institute of Electronics, Information and Communication Engineers of Japan.

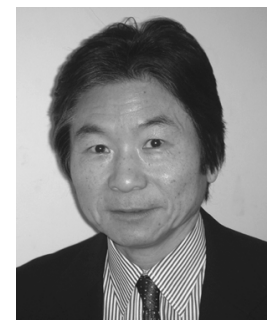

Hisamatsu Nakano (M'75-SM'87-F'92) was born in Ibaraki, Japan, on April 13, 1945. He received his B.E., M.E., and Dr.E. degrees in electrical engineering from Hosei University, Tokyo, in 1968, 1970 , and 1974, respectively.

Since 1973, he has been a member of the faculty of Hosei University, where he is now a Professor of electronic informatics. His research topics include numerical methods for low- and high-frequency antennas and optical waveguides. He has published more than 230 refereed journal papers, more than 270 international symposium papers, and more than 870 national symposium papers. He is the author of a book entitled Helical and Spiral Antennas (Research Studies Press, Wiley, 1987) and the co-author of Analysis Methods of Electromagnetic Wave Problems, Volume Two (Artech House, 1986). In addition, he is the author of Helical and Spiral Antennas, Encyclopedia of Communications (Wiley, 2002).

He received the IEE International Conference on Antennas and Propagation Best Paper Award and the IEEE Transactions on Antennas and Propagation Best Application Paper Award (H. A. Wheeler Award) in 1989 and 1994, respectively. In 1992, he was elected an IEEE fellow for contributions to the design of spiral and helical antennas. In 2001, he received the Award of Distinguished Technical Communication (from the Society for Technical Communication, USA) and the Science and Technology Progress Award (from Hangzhou, China).

Prof. Nakano is an associate editor of several journals and magazines, such as Electromagnetics, IEEE Antennas and Propagation Magazine, IEEE ANTENNAS AND WIRELESS PROPAGATION LETTERS, and Asian Information-Science-Life. 